\title{
Real-world renal function among patients with multiple myeloma in the United States
}

\author{
Joseph Mikhael $\mathbb{D}^{1}$, Erin Singh ${ }^{2}$ and Megan S. Rice ${ }^{2}$
}

\section{Dear Editor,}

Multiple myeloma (MM) is the second most common hematologic malignancy and is associated with substantial patient burden ${ }^{1}$. Despite the introduction of newer agents, patients with MM continue to experience relapses and/or become treatment refractory ${ }^{2}$. Renal impairment has been shown to affect up to $50 \%$ of patients with $\mathrm{MM}^{3}$, and has been reported to be an independent predictor of poor survival outcomes, with a median survival of approximately half that of MM patients without renal impairment ${ }^{4,5}$.

The aims of this analysis were to assess change in renal function by drug class (i.e., proteasome inhibitor [PI], immunomodulatory drug [IMiD], and monoclonal antibody $[\mathrm{mAb}]$ ) among patients with $\mathrm{MM}$ and renal impairment (defined as estimated glomerular filtration rate $[\mathrm{eGFR}]<50 \mathrm{~mL} / \mathrm{min} / 1.73 \mathrm{~m}^{2}$ using the Modification of Diet in Renal Disease equation [MDRD]) in early treatment lines, and to assess real-world patient outcomes by baseline renal status, renal response, and drug class.

This study used the nationwide Flatiron Health electronic health record-derived de-identified database. The Flatiron Health database is a longitudinal database, comprising de-identified patient-level structured and unstructured data, curated via technology-enabled abstraction $^{6,7}$. During the study period, the de-identified data originated from $\sim 280$ cancer clinics $(\sim 800$ sites of care). The study included 10,389 patients diagnosed with MM from January 1, 2011, to November 30, 2019. After excluding patients with probable missing treatment information, there were 6994 patients who received $\geq 1$ line of MM therapy and had information on race. Another four patients with unlikely creatinine levels (i.e., extremely

\footnotetext{
Correspondence: Joseph Mikhael (jmikhael@myeloma.org)

${ }^{1}$ Applied Cancer Research and Drug Discovery, Translational Genomics Research Institute, City of Hope Cancer Center, Phoenix, AZ 85004, USA ${ }^{2}$ Sanofi, Cambridge, MA 02142, USA
}

high or low) were excluded, resulting in 6990 patients (Supplemental Fig. 1).

eGFR-MDRD was calculated from creatinine lab values using the following equation: $175 \times$ (creatinine $\mathrm{mg} /$ $\mathrm{dL})^{-1.154} \times(\mathrm{age})^{-0.203} \times(0.742$ if female $) \times(1.212$ if Black/ African American $)^{8-10}$. The distribution of patients was assessed by eGFR-MDRD level $(<50$ and $\geq 50 \mathrm{~mL} / \mathrm{min} /$ $\left.1.73 \mathrm{~m}^{2}\right)$ at the start of first- $(1 \mathrm{~L})$ and second-line $(2 \mathrm{~L})$ therapy. Overall survival (OS) was evaluated by treatment line, stratified by eGFR-MDRD level at the start of the treatment line. Renal response was assessed in patients with eGFR-MDRD $<50 \mathrm{~mL} / \mathrm{min} / 1.73 \mathrm{~m}^{2}$ at the start of treatment, who had $\geq 1$ eGFR-MDRD measurement during the treatment line; using International Myeloma Working Group recommendations, patients with complete renal response (CRR) were defined as patients with $\geq 1$ eGFR-MDRD measurement $\geq 60 \mathrm{~mL} / \mathrm{min} / 1.73 \mathrm{~m}^{2}$ during the treatment line.

Logistic regression models were used to examine the association between treatment class and CRR status. These models were adjusted for the following clinically relevant variables, as they were potential confounders of the examined associations: other treatment classes received, age, sex, race, practice type, year of therapy line, and cytogenetic risk. Kaplan-Meier analyses and Cox proportional hazard models were used to examine OS from the start of $1 \mathrm{~L}$ therapy and $2 \mathrm{~L}$ therapy by baseline renal status and by both treatment class and renal response. Cox proportional hazard models were adjusted, as described above. The data that support the findings of this study have been originated by Flatiron Health, Inc. These de-identified data may be made available upon request, and are subject to a license agreement with Flatiron Health; interested researchers should contact DataAccess@flatiron.com to determine licensing terms. Flatiron Health, Inc, did not participate in the analysis of this data. 
Patient characteristics overall and by eGFR-MDRD level $\left(<50\right.$ and $\left.\geq 50 \mathrm{~mL} / \mathrm{min} / 1.73 \mathrm{~m}^{2}\right)$ at the start of $1 \mathrm{~L}$ therapy are shown in Supplemental Table 1. The mean age at start of initial therapy was 68.3 years, $45.8 \%$ of patients were female, and $17.2 \%$ were Black/African American. One quarter $(n=1722)$ of patients at the start of initial therapy had eGFR-MDRD $<50 \mathrm{~mL} / \mathrm{min} / 1.73 \mathrm{~m}^{2}$ (including $10.5 \%$ with eGFR-MDRD $<30 \mathrm{~mL} / \mathrm{min} / 1.73 \mathrm{~m}^{2}$ ), approximately half $(n=3465)$ had eGFR-MDRD $\geq 50 \mathrm{~mL} / \mathrm{min} / 1.73 \mathrm{~m}^{2}$, and the remainder had unknown levels. On average, patients with eGFR-MDRD $<50 \mathrm{~mL} / \mathrm{min} / 1.73 \mathrm{~m}^{2}$ at the start of initial therapy were older (71.3 vs 67.3 years) and more likely to be diagnosed at stage III (37.7\% vs $10.3 \%)$ compared with patients with eGFR-MDRD $\geq 50 \mathrm{~mL} / \mathrm{min} / 1.73 \mathrm{~m}^{2}$.

From the start of $1 \mathrm{~L}$ and $2 \mathrm{~L}$ therapy, patients with eGFR-MDRD $<50 \mathrm{~mL} / \mathrm{min} / 1.73 \mathrm{~m}^{2}$ had worse OS compared with those with eGFR-MDRD $\geq 50 \mathrm{~mL} / \mathrm{min} / 1.73 \mathrm{~m}^{2}$ ( $1 \mathrm{~L}$ median OS, 3.46 vs 5.89 years; $1 \mathrm{~L}$ unadjusted hazard ratio [HR], 1.79 [95\% confidence interval (CI), 1.63-1.97]; $2 \mathrm{~L}$ median OS, 2.67 vs 4.44 years; $2 \mathrm{~L}$ unadjusted HR, 1.70 [95\% CI, 1.52-1.91]; Supplemental Fig. 2). These differences remained statistically significant after adjustment for several factors, including age $(1 \mathrm{~L}$ multivariable-adjusted hazard ratio [aHR], 1.58 [95\% CI, 1.43-1.73]; 2 L multivariable aHR, 1.49 [95\% CI, 1.33-1.68]; Supplemental Table 2). Similar results were seen for patients with eGFR$\mathrm{MDRD}<30 \mathrm{~mL} / \mathrm{min} / 1.73 \mathrm{~m}^{2}$ compared with those with eGFR-MDRD $\geq 50 \mathrm{~mL} / \mathrm{min} / 1.73 \mathrm{~m}^{2}$ from the start of $1 \mathrm{~L}$ (multivariable aHR, 2.14; 95\% CI, 1.90-2.41) and $2 \mathrm{~L}$ therapy (multivariable aHR, 1.95; 95\% CI, 1.66-2.29).

Among all patients with eGFR-MDRD $<50 \mathrm{~mL} / \mathrm{min} /$ $1.73 \mathrm{~m}^{2}$ at the start of the treatment line and $\geq 1$ eGFRMDRD measurement during the treatment line $(N=1682$ in $1 \mathrm{~L}$ and 920 in $2 \mathrm{~L}$ ), $38.5 \%$ of patients achieved a CRR during $1 \mathrm{~L}$ therapy, but only $19 \%$ achieved a CRR during $2 \mathrm{~L}$ therapy. The majority of these patients with renal impairment had $1 \mathrm{~L}$ and $2 \mathrm{~L}$ use of PI $(77 \%$ and $64 \%$, respectively). These patients were significantly more likely to have a CRR compared with those without PI use in both $1 \mathrm{~L}$ (adjusted odds ratio [aOR], 1.36; 95\% CI, $1.04-1.77$ ) and $2 \mathrm{~L}$ therapy (aOR, 2.09; 95\% CI, 1.38-3.16) in multivariable-adjusted models.

Approximately half of patients with renal impairment had IMiD use in $1 \mathrm{~L}$ and $2 \mathrm{~L}$ therapy. In both treatment lines, patients with IMiD use were significantly more likely to have a CRR compared with those without IMiD use in multivariable models, particularly in $1 \mathrm{~L}$ therapy (aOR, 2.14; 95\% CI, 1.68-2.72).

When we examined PI and IMiD use in combination together among patients with renal impairment, $36 \%$ of patients had combination PI and IMiD treatment, $42 \%$ used PIs but not IMiDs, $14 \%$ used IMiDs but not PIs, and $8 \%$ used neither in $1 \mathrm{~L}$ therapy. In $2 \mathrm{~L}, 26 \%$ of patients had

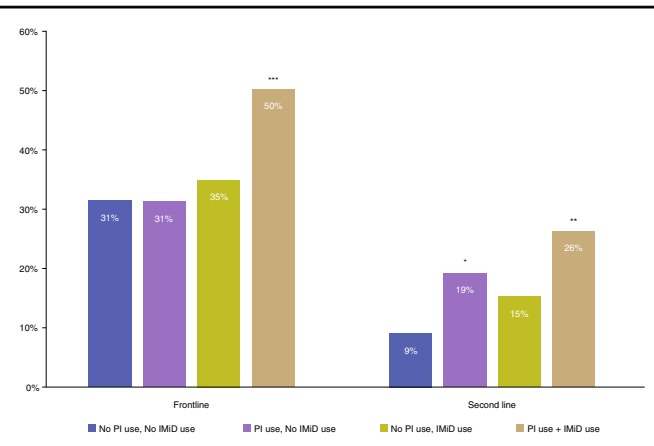

Fig. 1 Complete renal response (CRR) by combined proteasome inhibitor (PI) use and immunomodulatory drug (IMiD) use in frontline and second line. Logistic regression models were used to examine the association between treatment class (i.e., PI and IMiD) and CRR status adjusted for other treatment classes received, age, sex, race, practice type, year of therapy line, and cytogenetic risk. ${ }^{*} P<0.05$, ${ }^{*} P<0.01,{ }^{* * *} P<0.001$ vs no PI or IMiD use from multivariable models. Multivariable models adjusted for other treatment classes received, age, sex, race, practice type, year of therapy line, and cytogenetic risk.

combination PI and IMiD treatment, 38\% used PIs but not IMiDs, $26 \%$ used IMiDs but not PIs, and $10 \%$ used neither. In $1 \mathrm{~L}$ and $2 \mathrm{~L}$ therapy, patients with both PI and IMiD use were significantly more likely to have a CRR compared with those without use of either treatment class (1 L aOR, 2.35 [95\% CI, 1.54-3.60]; $2 \mathrm{~L}$ aOR, 3.89 [95\% CI, $1.71-8.86])$. In $2 \mathrm{~L}$ therapy, patients with PI use but no IMiD use also were significantly more likely to have a CRR compared with those without use of either (aOR, 2.54; 95\% CI, 1.15-5.59; Fig. 1). Patients with eGFR-MDRD < $30 \mathrm{~mL} / \mathrm{min} / 1.73 \mathrm{~m}^{2}$ with both PI and IMiD use were significantly more likely to have a CRR compared with those without the use of either treatment class in $1 \mathrm{~L}$ (aOR, 5.46; 95\% CI, 1.77-16.8), but there was no association in $2 \mathrm{~L}$ (aOR, 0.50; 95\% CI, 0.06-4.17).

Low mAb use in early treatment lines during the database time frame (through November 2019) prevented most analyses of this treatment class. Few patients $(<1 \%)$ were treated with $\mathrm{mAb}$ frontline and $\sim 10 \%$ had $\mathrm{mAb}$ use as $2 \mathrm{~L}$ therapy. We did not observe a significant difference in CRR by mAb use in $2 \mathrm{~L}$ ( $22 \%$ vs $19 \%$; aOR, 1.51 ; $95 \%$ CI, $0.84-2.74)$. Due to small sample sizes, we were unable to examine $\mathrm{mAb}$ combination therapies.

Patients receiving combination PI and IMiD treatment who had a CRR had significantly better OS compared with patients without use of either treatment, who did not have a CRR in both frontline (aHR, 0.52; 95\% CI, 0.37-0.73) and second line (aHR, 0.53; 95\% CI, 0.32-0.88; Supplemental Table 3). This relationship was not observed for patients with eGFR-MDRD $<30 \mathrm{~mL} / \mathrm{min} / 1.73 \mathrm{~m}^{2}$; however, due to the relatively smaller sample size, we cannot make definitive conclusions for this subgroup. 
In this study, MM patients with renal impairment were more likely to be older and to present with ISS stage III at diagnosis, which has been reported in a previous phase III study ${ }^{11}$. Patients with renal impairment exhibited decreased OS compared with non-renally impaired patients. Patients treated with combination therapy that included PIs and IMiDs together in early treatment lines were more likely to have a CRR, supporting the known benefits of triplet vs doublet therapy. In addition, OS was prolonged among patients with PI and IMiD combination therapy who achieved a CRR, highlighting the benefits of both combination therapy and renal response. Moreover, these data underscore the importance of providing patients the best therapy for their MM.

This study has some limitations. We were unable to discern whether renal impairment was due to MM or other causes. Though we adjusted for several baseline characteristics, including age and practice type, we may not have fully accounted for factors influencing receipt of triplet regimens. In addition, as few patients received mAbs or had very low renal function (eGFR-MDRD $<30 \mathrm{~mL} / \mathrm{min} / 1.73 \mathrm{~m}^{2}$ ) in early treatment lines, power for analyses in these subgroups was limited. The primary strengths of this study are the use of repeated eGFR-MDRD assessments and adjustment for several potential confounders.

MM patients with renal insufficiency have been shown to have poorer outcomes and continue to have unmet medical need. Overall, these data suggest that using combination therapy early with the goal of inducing a renal response in these patients is not only feasible, but also may result in improved outcomes. Future investigations with larger datasets that include newer agents may improve the understanding of the optimal combination treatment regimens for these patients.

\section{Acknowledgements}

The authors acknowledge medical writing assistance from Erin Burns-Tidmore, Ph.D., of Elevate Medical Affairs, contracted by Sanofi Genzyme for publication support services. The authors also thank Sarah Naeger, Ph.D., M.P.H., for her contributions. Sources of funding: Sanofi.

\section{Author contributions}

All authors contributed to the design of the study. M.S.R. performed the analyses with input from J.M. and E.S. All authors contributed to interpretation of the data. All authors revised the work for important intellectual content and assume responsibility for data integrity and the decision to submit this manuscript for publication; had full access to the study data; edited and reviewed manuscript drafts; and approved the final version for submission.

\section{Conflict of interest}

J.M.: Honoraria: Amgen, Celgene, GlaxoSmithKline, Janssen Pharmaceuticals, Karyopharm Therapeutics, Sanofi, and Takeda Pharmaceutical Company. E.S. and M.S.R.: employed by Sanofi, and may hold stock and/or stock options in the company.

\section{Publisher's note}

Springer Nature remains neutral with regard to jurisdictional claims in published maps and institutional affiliations.

Supplementary information The online version contains supplementary material available at https://doi.org/10.1038/s41408-021-00492-6.

Received: 22 February 2021 Revised: 28 April 2021 Accepted: 5 May 2021 Published online: 21 May 2021

\section{References}

1. Moreau, P. et al. Multiple myeloma: ESMO Clinical Practice Guidelines for diagnosis, treatment and follow-up. Ann. Oncol. 28, iv52-iv61 (2017).

2. Rajkumar, S. V. Multiple myeloma: 2016 update on diagnosis, risk-stratification, and management. Am. J. Hematol. 91, 719-734 (2016).

3. Clark, A. D., Shetty, A. \& Soutar, R. Renal failure and multiple myeloma: pathogenesis and treatment of renal failure and management of underlying myeloma. Blood Rev. 13, 79-90 (1999).

4. Eleutherakis-Papaiakovou, V. et al. Renal failure in multiple myeloma: incidence, correlations, and prognostic significance. Leuk. Lymphoma 48, 337-341 (2007).

5. San-Miguel, J. F. et al. Efficacy and safety of bortezomib in patients with renal impairment: results from the APEX phase 3 study. Leukemia 22, 842-849 (2008).

6. Ma, X., Long, L., Moon, S., Adamson, B. J. S. \& Baxi, S. S. Comparison of population characteristics in real-world clinical oncology databases in the US: Flatiron Health, SEER, and NPCR. Preprint at medRxiv https://doi.org/10.1101/ 2020.03.16.20037143 (2020).

7. Birnbaum, B. et al. Model-assisted cohort selection with bias analysis for generating large-scale cohorts from the EHR for oncology research. Preprint at https:/arxiv.org/abs/2001.09765 (2020).

8. Levey, A. S. et al. Expressing the Modification of Diet in Renal Disease Study equation for estimating glomerular filtration rate with standardized serum creatinine values. Clin. Chem. 53, 766-772 (2007).

9. Levey, A. S. et al. Using standardized serum creatinine values in the modification of diet in renal disease study equation for estimating glomerular filtration rate. Ann. Intern. Med. 145, 247-254 (2006).

10. Stevens, L. A. et al. Impact of creatinine calibration on performance of GFR estimating equations in a pooled individual patient database. Am. J. Kidney Dis. 50, 21-35 (2007).

11. Dimopoulos, M. A. et al. Isatuximab plus pomalidomide and dexamethasone in relapsed/refractory multiple myeloma patients with renal impairment: ICARIA-MM subgroup analysis. Leukemia 35, 562-572 (2021). 